\title{
The Study of Westward Drift in the Main Geomagnetic Field
}

\author{
G. Bayanjargal \\ Research Center for Astronomy and Geophysics, Mongolian Academy of Sciences (MAS), Ulaanbaatar, Mongolia \\ Correspondence should be addressed to G. Bayanjargal; readersman@yahoo.com
}

Received 8 January 2013; Revised 12 March 2013; Accepted 20 March 2013

Academic Editor: Rudolf A. Treumann

Copyright (C) 2013 G. Bayanjargal. This is an open access article distributed under the Creative Commons Attribution License, which permits unrestricted use, distribution, and reproduction in any medium, provided the original work is properly cited.

\begin{abstract}
We have obtained a solution for the velocity of westward drift from the induction equation in which an approach for main geomagnetic field was built. Distribution functions $B(r, t)$ entered into the induction equation have been built by the observatories' data in North America and the Europe from 1991 to 2006. The longitudinal -0.123 degree/year and latitudinal 0.068 degree/year drifts were defined in North America. And the longitudinal - 0.257 degree/year drift was defined in Europe from 1991 to 2006 . These drifts are similar to results of other studies.
\end{abstract}

\section{Introduction}

Westward drift in the main geomagnetic field (MGF) has been studied since discovered by Halley [1]. We could write it briefly from the previous results of investigations by other people. Bauer [2] studied the drift of the zero line of declination tracing. He detected that the mean velocity was $-0.22 \mathrm{deg} / \mathrm{yr}$. Bullard et al. [3] also studied the nondipole field at epoch 1907.5 and 1945. They determined that the drift velocity was $-0.26 \mathrm{deg} / \mathrm{yr}$. Yukutake [4] estimated that the mean value of westward drift was $-0.20 \mathrm{deg} / \mathrm{yr}$ in $1850-1950$. Wei and $\mathrm{Xu}$ [5] estimated that the mean value of westward drift was $-0.18 \mathrm{deg} / \mathrm{yr}$ in $1900-2000$. These researches were based mainly on the spherical harmonic potential's formula for main geomagnetic field.

If we have got the geomagnetic field's distribution function of space and time, can we define the westward drift on the Earth's little area such as a continent or any part of continents by other method? Is there any theoretical possibility to decide this problem? When I read Yukutake's paper of 1962, the spirit of solving the previous problem arose to me. Our task is to answer these questions or to find a new method. Yukutake [4] hinted an idea to write a similar equation with the induction equation written in liquid core for Earth's surface. But Yukutake did not estimate the westward in MGF from the induction equation. He studied the westward in MGF by scalar potential's method.
The westward drift in MGF is the result of interaction between fluid motions on the core mantle boundary $\mathrm{CMB}$ and the strong toroidal field. And the westward drift is observed in steady MGF in a large interval of time scale. The effects of fluid motions with small scales of space and time on CMB are not observed on the Earth's surface because these effects could be decreased by mantle.

Thus, we have built an approach for the induction equation on $\mathrm{CMB}$ by mean field's dynamic theory. And we have obtained the form of the induction equation for Earth's surface.

I think that the westward drift in MGF was not obtained from the induction equation in the past. The reason is that many satellites have been blasted in orbital paths around the Earth since 1962. And scalar potential's method for MGF has been rapidly developed since 1962 . Thus, the idea of studying the westward drift in MGF by the induction equation has been forgotten since 1962.

In this paper, we have studied the westward drift in MGF by the induction equation. And we have used the geomagnetic field's distribution functions of space and time in North America and Europe, because internet network had been built since around 1980. And http://intermagnet.org/ had been built since 1990. Therefore, we could build $B(r, t)$ distribution functions of space and time by data of http://intermagnet.org/ in Europe and North America. These 
distribution functions have been defined by the data of observatories in Figures 1 and 2.

\section{The Approach in the Induction Equation for Main Geomagnetic Field}

Strong toroidal field is generated by Herndon's postulate [6] in subshell of georeactor in the Earth's inner core. The strong toroidal field is steady field. In other words, the energy of the strong toroidal field does not change in a few years. The strong toroidal field generated in the center of inner core spreads through the inner core, liquid core, and mantle. And it is observed as the main geomagnetic field (MGF) on the Earth's surface.

Most effectual movements of electrical conduction substances, which are interacted with the strong toroidal field, exist only in the Earth's liquid core.

The Earth's liquid core is about 23 percent denser at the bottom than at top. This difference of density blocks the possibility that radial flows are generated by the thermal convection in Earth's liquid. Thus, thermal equilibrium does not establish for whole Earth's liquid core. And the flows in Earth's liquid move in a horizontal direction. In other words, the radial component of the flow is very small in the Earth's liquid core. And pressure increases in the direction of depth of the liquid core. The fluidity of liquid core also decreases in the direction of depth.

Thus, the following representation exists in the geodynamic theory. The most effectual flows or fluid motions, which interact with the strong toroidal field, exist on the core mantle boundary $(\mathrm{CMB})$. The interaction between the fluid motions on the $\mathrm{CMB}$ and the strong toroidal field produces irregular magnetic fields with small scale. The irregular magnetic fields are called non-dipole part of the MGF. And variation with the long time period exists in the non-dipole part of the MGF. The variation of MGF is called secular variation (SV). On the Earth's surface, the westward drift in the MGF is also observed by the influence of the fluid motions on the CMB. The interactions between the fluid motions on the $\mathrm{CMB}$ and the strong toroidal field are studied by the governing differential equation.

The governing differential equation is written in the form

$$
\frac{d \vec{B}}{d t}=\vec{\nabla} \times(\vec{V} \times \vec{B})+\eta \nabla^{2} \vec{B} .
$$

Equation (1) is called the induction equation. Here, $\eta$ is the coefficient of magnetic diffusivity and $\vec{V}$ is the local velocity of the conductive fluid motions on the CMB.

It is possible that the fluid motions on the $\mathrm{CMB}$ are divided into two components such as mean motions and turbulent motions. Velocity of the mean motions is steady and its $L$-scale is long. $l$-scale of the turbulent motions is small as $L \gg l$. The velocity of the turbulent motions rapidly changes and it has fluctuations. They are written in the form

$$
\begin{aligned}
& \vec{V}=\vec{V}_{0}+\vec{v}=\text { mean + fluctuations, } \\
& \vec{B}=\vec{B}_{0}+\vec{b}=\text { mean + fluctuations. }
\end{aligned}
$$

These effects of fluctuations on the mean field on CMB are very small. They are also decreased by mantle. Thus, the effects of fluctuations are mostly not observed in the main geomagnetic field on the Earth's surface.

Therefore, (1) must be averaged when (2) is substituted into it. Equation (1) is averaged by (3), called Reynold's rules for averaging, which are

$$
\begin{gathered}
\left\langle\vec{V}_{0}\right\rangle=\vec{V}_{0}, \quad\langle\vec{v}\rangle=0, \\
\langle\vec{V}\rangle=\left\langle\vec{V}_{0}+\vec{v}\right\rangle=\left\langle\vec{V}_{0}\right\rangle+\langle\vec{v}\rangle=\vec{V}_{0}, \\
\left\langle\vec{B}_{0}\right\rangle=\vec{B}_{0}, \quad\langle\vec{b}\rangle=0, \\
\langle\vec{B}\rangle=\left\langle\vec{B}_{0}+\vec{b}\right\rangle=\left\langle\vec{B}_{0}\right\rangle+\langle\vec{b}\rangle=\vec{B}_{0} .
\end{gathered}
$$

where $\langle\cdots\rangle$ shows statistical averaging, $(\approx$ volume or time averaging). The substitution and averaging of (1), (2), and (3) are shown as follows:

$$
\begin{aligned}
\left\langle\frac{d \vec{B}_{0}}{d t}+\frac{d \vec{b}}{d t}\right\rangle= & \vec{\nabla} \times\left\langle\left(\vec{V}_{0}+\vec{v}\right) \times\left(\vec{B}_{0}+\vec{b}\right)\right\rangle \\
& +\eta \nabla^{2}\left\langle\vec{B}_{0}+\vec{b}\right\rangle .
\end{aligned}
$$

Equation (4) can be written in the following form:

$$
\frac{d \vec{B}_{0}}{d t}=\vec{\nabla} \times\left\langle\left(\vec{V}_{0}+\vec{v}\right) \times\left(\vec{B}_{0}+\vec{b}\right)\right\rangle+\eta \nabla^{2} \vec{B}_{0} .
$$

The term $\vec{\nabla} \times\left\langle\left(\vec{V}_{0}+\vec{v}\right) \times\left(\vec{B}_{0}+\vec{b}\right)\right\rangle$ in (5) can be written in the expanded form

$$
\begin{aligned}
\vec{\nabla} \times & \left\langle\left(\vec{V}_{0}+\vec{v}\right) \times\left(\vec{B}_{0}+\vec{b}\right)\right\rangle \\
= & \vec{\nabla} \times\left(\left\langle\vec{V}_{0} \times \vec{B}_{0}\right\rangle+\left\langle\vec{V}_{0} \times \vec{b}\right\rangle\right. \\
& \left.+\left\langle\vec{v} \times \vec{B}_{0}\right\rangle+\langle\vec{v} \times \vec{b}\rangle\right) \\
= & \vec{\nabla} \times\left(\vec{V}_{0} \times \vec{B}_{0}\right)+\vec{\nabla} \times\langle\vec{v} \times \vec{b}\rangle .
\end{aligned}
$$

The terms $\left\langle\vec{V}_{0} \times \vec{b}\right\rangle=\vec{V}_{0} \times\langle\vec{b}\rangle=0$ and $\left\langle\vec{v} \times \vec{B}_{0}\right\rangle=\langle\vec{v}\rangle \times \vec{B}_{0}=0$ in (6) are vanished by Reynold's rules. When (6) is substituted into (5), it will be like

$$
\begin{aligned}
\frac{d \vec{B}_{0}}{d t}= & \vec{\nabla} \times\left(\vec{V}_{0} \times \vec{B}_{0}\right) \\
& +\vec{\nabla} \times\langle\vec{v} \times \vec{b}\rangle+\eta \nabla^{2} \vec{B}_{0} .
\end{aligned}
$$

Equation (7) is called the averaged induction equation. The term $\vec{\nabla} \times\langle\vec{v} \times \vec{b}\rangle$ in (7) expresses the effects of turbulence on the mean magnetic field. The Equation

$$
\vec{\varepsilon}=\langle\vec{v} \times \vec{b}\rangle
$$

is called the mean electromotive force. There is a linear relation between the mean electromotive force $\vec{\varepsilon}$ and 


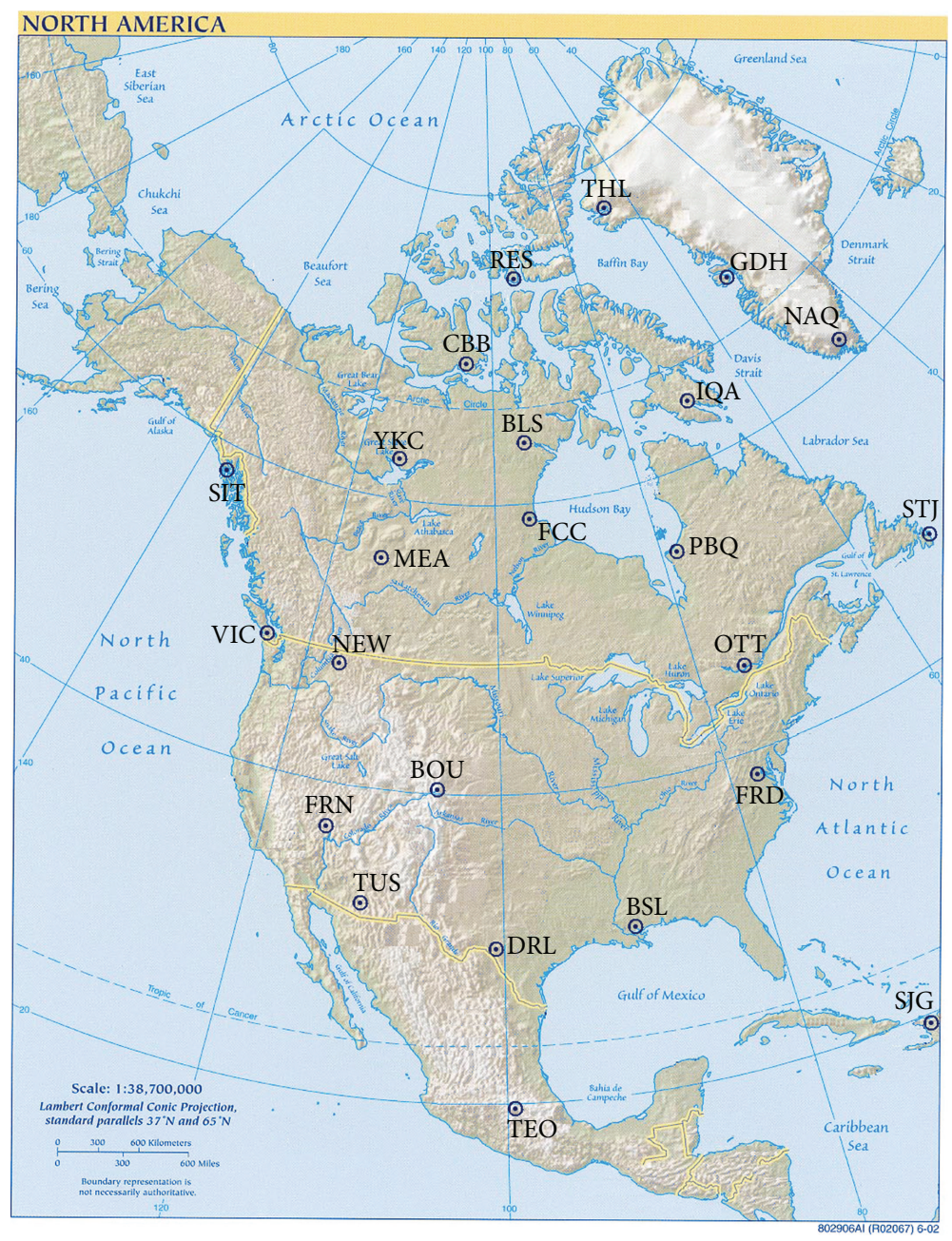

FIgURE 1: The locations of the observatories in North America.

the mean magnetic field $\vec{B}_{0}$. The relation is expressed in the following equation:

$$
\begin{gathered}
\vec{\varepsilon}=\alpha \vec{B}_{0}-\beta \vec{\nabla} \times \vec{B}_{0}+\cdots, \\
\alpha=-\frac{1}{3}\langle\vec{v} \cdot(\vec{\nabla} \times \vec{v})\rangle \tau, \\
\beta=\frac{1}{3} v^{2} \tau
\end{gathered}
$$

called Taylor's expansion. Here, $\alpha$ is the coefficient of the helical effects of fluid motion on the $\mathrm{CMB}, \beta$ is the coefficient for the turbulent diffusivity, and $\tau$ is lifetime of the helixes.

When (9) is substituted into (7), it becomes

$$
\begin{aligned}
\frac{d \vec{B}_{0}}{d t}= & \vec{\nabla} \times\left(\vec{V}_{0} \times \vec{B}_{0}\right) \\
& +\vec{\nabla} \times \alpha \vec{B}_{0}+(\eta+\beta) \nabla^{2} \vec{B}_{0} .
\end{aligned}
$$

The resulting equation (10) is the governing equation of the mean field on the CMB.
After expanding the term as $\vec{\nabla} \times\left(\vec{V}_{0} \times \vec{B}_{0}\right)=-\left(\vec{V}_{0} \cdot \vec{\nabla}\right) \vec{B}_{0}+$ $\left(\vec{B}_{0} \cdot \vec{\nabla}\right) \vec{V}_{0}-\vec{B}_{0}\left(\vec{\nabla} \cdot \vec{V}_{0}\right)$ in $(10)$, it becomes

$$
\begin{aligned}
\frac{d \vec{B}_{0}}{d t}= & -\left(\vec{V}_{0} \cdot \vec{\nabla}\right) \vec{B}_{0}+\left(\vec{B}_{0} \cdot \vec{\nabla}\right) \vec{V}_{0} \\
& -\vec{B}_{0}\left(\vec{\nabla} \cdot \vec{V}_{0}\right)+\vec{\nabla} \times \alpha \vec{B}_{0} \\
& +(\eta+\beta) \nabla^{2} \vec{B}_{0},
\end{aligned}
$$

where $-\left(\vec{V}_{0} \cdot \vec{\nabla}\right) \vec{B}_{0}$ is advection term, $\left(\vec{B}_{0} \cdot \vec{\nabla}\right) \vec{V}_{0}$ is stretching term, $-\vec{B}_{0}\left(\vec{\nabla} \cdot \vec{V}_{0}\right)$ is compression term, and $(\eta+\beta) \nabla^{2} \vec{B}_{0}$ is diffusion and decay term. $\vec{V}_{0}$ is the mean velocity of the conductivity of the fluid motion on the CMB.

Equation (11) can be written in the form

$$
\begin{aligned}
\frac{d \vec{B}_{0}}{d t} & +\left(\vec{V}_{0} \cdot \vec{\nabla}\right) \vec{B}_{0} \\
= & \left(\vec{B}_{0} \cdot \vec{\nabla}\right) \vec{V}_{0}-\vec{B}_{0}\left(\vec{\nabla} \cdot \vec{V}_{0}\right) \\
& +\vec{\nabla} \times \alpha \vec{B}_{0}+(\eta+\beta) \nabla^{2} \vec{B}_{0} .
\end{aligned}
$$




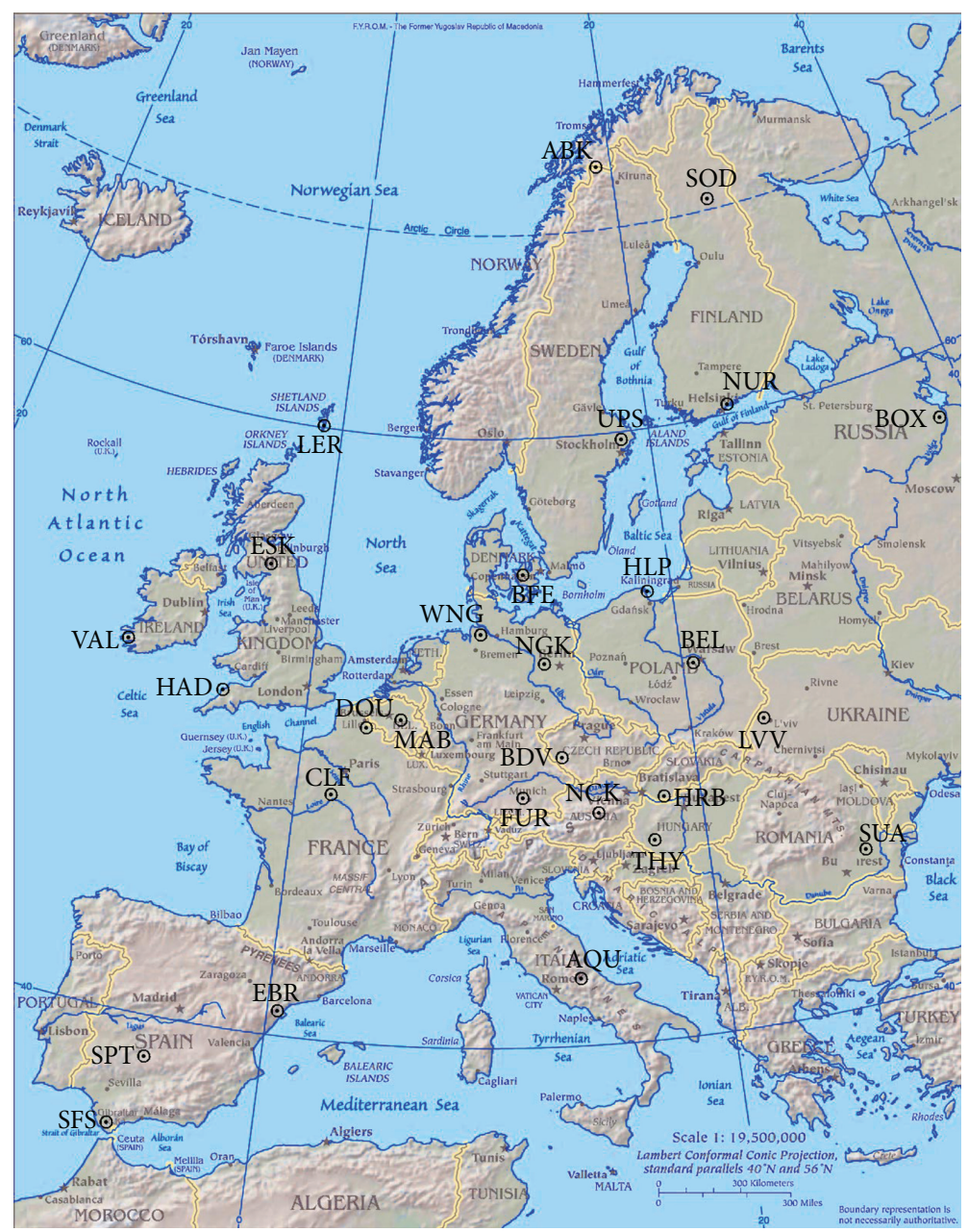

FIgURE 2: The locations of observatories in Europe.

Generally, $\vec{V}_{0}$ is steady on the CMB and its local variation is very small. Thus, the effects of the terms $\left(\vec{B}_{0} \cdot \vec{\nabla}\right) \vec{V}_{0}$ and $-\vec{B}_{0}(\vec{\nabla}$. $\vec{V}_{0}$ ) on (12) for the CMB are very small. In other words, there are almost no stretching and the compression or we can say that they do not exist in the conductivity of the fluid motion on the $\mathrm{CMB}$

The terms $\vec{\nabla} \times \alpha \vec{B}_{0}$ and $\beta \nabla^{2} \vec{B}_{0}$ in (12) show turbulent effects in the fluid motion on the CMB. The scales of $\alpha$ and $\beta$ are small. The life-time $\tau$ in $\alpha$ and $\beta$ in (9) is also small. Therefore, the effects of the terms may be observed around one point or one observatory on the Earth's surface during short time. But the effects of the terms with $\alpha$ and $\beta$ are small in the large interval of space and time. The reason is that the effects of these terms are decreased when they are integrated by the large interval of space and time. For example, the distribution functions of $B x, B y$, and $B z$ had covered continents such as Europe and North America in 1991-2006. Thus, the influence of the terms with $\alpha$ and $\beta$ is very small in the same equation as (12) that will be written for the surface of the Earth or in the mantle.

As aftermentioned, (12) is only valid for the liquid core. But it can be seen from (12) that the advection term is the main factor in the secular variation of MGF on the Earth's surface.

We can write an equation of the same kind with (12) on the Earth's surface. The equation is

$$
\frac{d \vec{B}}{d t}+\left(\vec{v}_{0}^{\prime} \cdot \vec{\nabla}\right) \vec{B}=\vec{R}(\vec{r}, t)
$$

where $\vec{r}$ denotes the positional vector and $\vec{v}_{0}^{\prime}$ is the drift velocity of the field on the Earth's surface or in the mantle. $\vec{R}(\vec{r}, t)$ is the residual field after the westward drift has been subtracted from the observed time variation. $\vec{R}(\vec{r}, t)$ can also be described as the right-hand side of (12). Because the influence of these terms is decreased by mantle. The effects of these terms on the residual field in (13) are observed as weaker than those in (12) on the Earth's surface.

Therefore, it is seen from (12)-(13) that the variation of MGF on the Earth's surface depends on the mean velocity of the conductivity of the fluid motion on the CMB.

Now, we can rewrite the total energy of the residual field in the form

$$
E=\int[\vec{R}(\vec{r}, t)]^{2} d s
$$


where $d s$ is an interval of space and time, $\vec{R}(\vec{r}, t)$ is a residual field, $[\vec{R}(\vec{r}, t)]^{2}$ is a variance of the energy density of the residual field in the small time interval, and $E$ is the energy of the residual field.

The energy of the residual field weakly depends on the same terms as the right-hand side of (12). The energy variation of the strong toroidal field sourced in subshell in the Earth's inner core is also very small and it equals to zero in a time of 20 years.

Therefore, the energy variation of the residual field equals to zero in a time of 20 years as

$$
\delta E=0 .
$$

Once we know the field distribution $\vec{B}$ and its time derivative $d \vec{B} / d t$, we would be able to find the velocity $\vec{v}_{0}^{\prime}$ from the condition that the energy variation of the residual field equals to zero on (15).

In this paper, we have solved the drift velocity $\vec{v}_{0}^{\prime}$ of field from the condition in (15).

\section{Construction of the Distribution Functions of Main Geomagnetic Field}

In this paper, we have studied the westward drift in the MGF on the territories of North America and Europe. We have the geomagnetic field's data of 1991-2006 in the observatories located in North America and Europe. The locations and the territories of the observatories are shown in Figures 1 and 2.

One-minute data of $X, Y$, and $Z$ in observatories in Figures 1 and 2 are used in this study. The components of the geomagnetic field can be displayed as Figure 3. Figure 3 is called the magnetogram.

The data of geomagnetic field noted in the observatories are the summary field that can be expressed as

$$
B=M+m+D+N+S+L
$$

where $M$ is the MGF, $m$ is the secular variation of MGF, and $D$ is a regular and irregular part of the disturbance field that is sourced outer from the Earth. $N$ is a long period field and it has no frequency that is sourced outer from the Earth. $N$ is also observed on the geomagnetic quiet days and it is not frequently observed. $S$, sourced by the solar effects, is the periodic field. The solar magnetic variation in the quiet days is denoted by Sq. $L$, sourced by the lunar effects, is periodic field. The term $M+m$ or MGF is about 90 percent of the summary intensity of magnetic field in (16). And the term $D+N+S+L$ is about 10 percent of the summary intensity.

Our aim is to subtract the term $M+m$ from the summary magnetic field in (16). We use a relatively simple method. The reason is that the maximum and minimum values of amplitude of any variation locate in a periodic time interval. We have averaged the geomagnetic field noted in observatories by (17). When the values of the amplitude in one period have been averaged, the maximum and minimum values are compensated. And the variations are decreased.
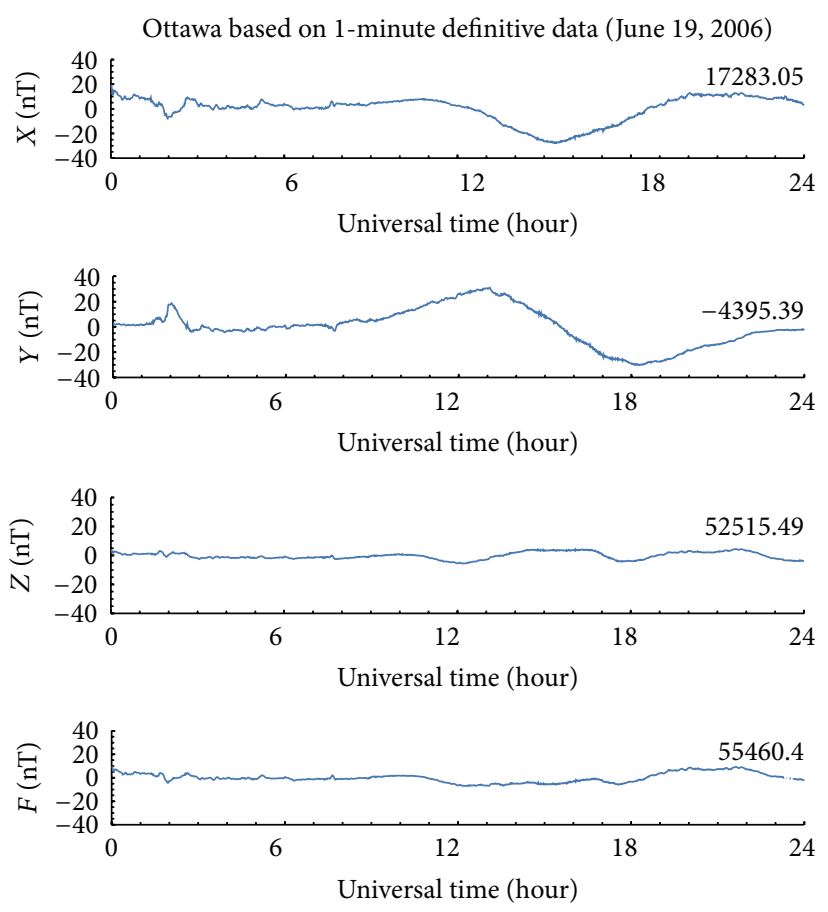

FIgURE 3: The magnetogram of a day in OTT.

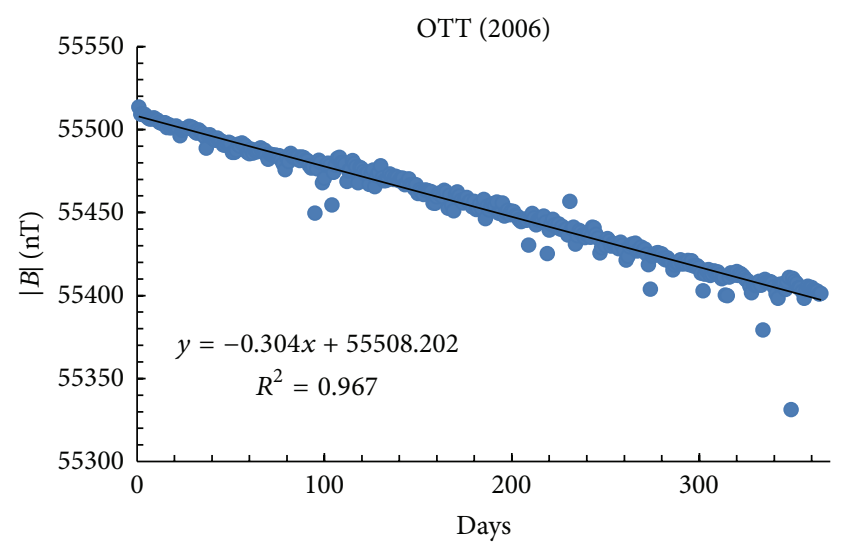

FIGURE 4: The dependence of the averaged value of MGF on the day order for OTT in 2006.

Thus, variations, period which is shorter than 24 hours, are decreased by the equation

$$
\bar{B}_{i}=\frac{\sum_{j=1}^{1440} B_{i j}}{1440}
$$

where $i$ is the number of days, $j$ is the minutes, $B_{i j}$ is the value of geomagnetic field noted in observatory in one minute, and $\bar{B}_{i}$ is the mean value of geomagnetic field for $i$ th day.

But variation $D$ is still there in the averaged values. Generally, the figure of the secular variation $m$ is a monotonic and regular function. We can depict the general tendency of the secular variation by plotting the averaged values in (17). The depiction is in Figure 4. 
We have also managed to subtract some days with great fluctuation by controlling them in the equation

$$
\sigma_{i}=\left(B(t)-\bar{B}_{i}\right)^{2}
$$

where $\sigma_{i}$ is the square of deviation of $i$ th day from the general tendency for one year. $B(t)$ is the general tendency for one year detected by the mean values of the days. $\bar{B}_{i}$ is the mean value for $i$ th day obtained using (17).

Generally, the effects of the magnetic storm are in the subtracted days. The effects of the magnetic storm have been eliminated by (18).

We have defined the mean geomagnetic field for a year by the equation

$$
\bar{B}=\frac{\sum_{j=1}^{N} \bar{B}_{i}}{N},
$$

where $\bar{B}$ is the mean geomagnetic field for a year, $\bar{B}_{i}$ is the mean value of the magnetic field for the remaining days, and $N$ is the number of the remaining days.

Variations with short period, such as minutes, days, months, and seasons, are decreased and eliminated by the previous averaging method. Thus, the MGF and its secular variation are in the mean value of the geomagnetic field for one year. The reason is that the previous averaging method cannot decrease or eliminate the secular variation with long period. Therefore, the mean values in (19) can represent the MGF and its secular variation.

We have defined the general tendency of the secular variation of MGF in the observatories in Figures 1 and 2.

Generally, it is seen that the secular variation of MGF in North America linearly decreases from 1991 to 2006. But in Europe, it linearly increases in the same time. Therefore, it can be considered that they are governed by one general dynamics.

We have defined the distribution functions of the MGF for each component in North America and Europe for each year. They are expressed by the polynomial regression which is

$$
\begin{aligned}
B x= & C_{00}+C_{01} \varphi+C_{02} \varphi^{2}+C_{03} \varphi^{3} \\
& +C_{10} \theta+C_{11} \theta \varphi+C_{12} \theta \varphi^{2} \\
& +C_{20} \theta^{2}+C_{21} \varphi \theta^{2}+C_{30} \varphi^{3}, \\
B y= & A_{00}+A_{01} \varphi+A_{02} \varphi^{2}+A_{03} \varphi^{3} \\
& +A_{10} \theta+A_{11} \theta \varphi+A_{12} \theta \varphi^{2} \\
& +A_{20} \theta^{2}+A_{21} \varphi \theta^{2}+A_{30} \theta^{3}, \\
B z= & K_{00}+K_{01} \varphi+K_{02} \varphi^{2}+K_{03} \varphi^{3} \\
& +K_{10} \theta+K_{11} \theta \varphi+K_{12} \theta \varphi^{2} \\
& +K_{20} \theta^{2}+K_{21} \varphi \theta^{2}+K_{30} \theta^{2},
\end{aligned}
$$

where $B x, B y$, and $B z$ are the components of MGF in the coordinate system NED, $\varphi$ is longitude, $\theta$ is latitude, and

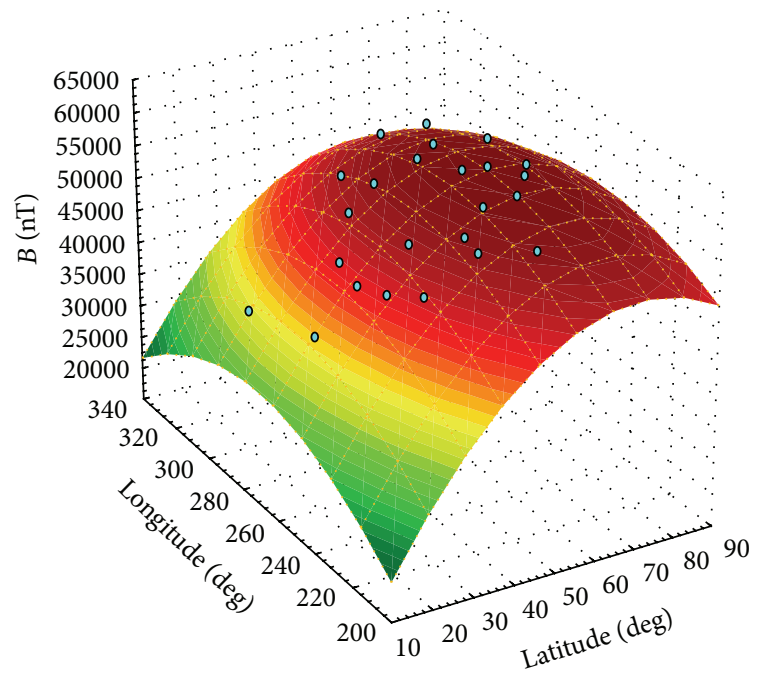

FIgURE 5: The MGF distribution functions in North America in 2006.

$C_{00}, \ldots, C_{30}, A_{00}, \ldots, A_{30}$, and $K_{00}, \ldots, K_{30}$ are polynomial coefficients, and

$$
\begin{aligned}
B x= & D_{00}+D_{01} \varphi+D_{02} \varphi^{2}+D_{03} \varphi^{3} \\
& +D_{10} \theta+D_{11} \theta \varphi+D_{12} \theta \varphi^{2} \\
& +D_{20} \theta^{2}+D_{21} \varphi \theta^{2}+D_{30} \theta^{3}, \\
B y= & Q_{00}+Q_{01} \varphi+Q_{02} \varphi^{2}+Q_{03} \varphi^{3} \\
& +Q_{10} \theta+Q_{11} \theta \varphi+Q_{12} \theta \varphi^{2} \\
& +Q_{20} \theta^{2}+Q_{21} \varphi \theta^{2}+Q_{30} \theta^{3}, \\
B z= & H_{00}+H_{01} \varphi+H_{02} \varphi^{2}+H_{03} \varphi^{3} \\
& +H_{10} \theta+H_{11} \theta \varphi+H_{12} \theta \varphi^{2} \\
& +H_{20} \theta^{2}+H_{21} \varphi \theta^{2}+H_{30} \theta^{3},
\end{aligned}
$$

where $D_{00}, \ldots, D_{30}, Q_{00}, \ldots, Q_{30}$, and $H_{00}, \ldots, H_{30}$ are polynomial coefficients.

For example, we have displayed the distribution functions of the MGF in Figures 5 and 6.

The form of the distribution functions of space and time is not important. And the confidence level of the formula must be of high percentage. In case the relative errors of the extracted values of MGF are smaller than $1 \%-5 \%, P$ values of the distribution functions in (20) and (21) are $95 \%-98 \%$ by the reduced chi-square test.

The polynomial coefficients in (20)-(21) for each year were defined using the least square method.

Afterwards, we have defined the dependence of the polynomial coefficients on time. They are written in (B.1)(B.3) and (B.4)-(B.6) in Appendix B.

We now have the distribution functions of MGF that depend on the space and time for North America and 


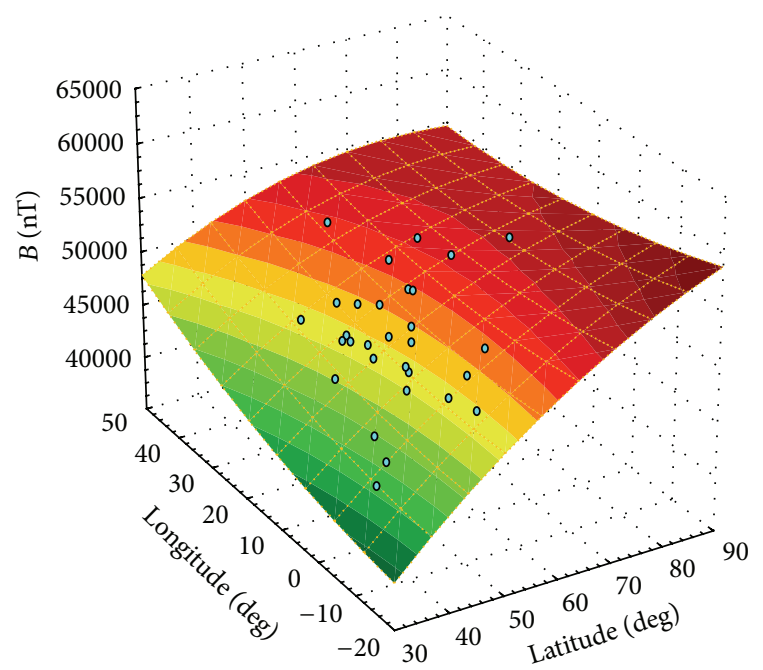

FIGURE 6: The MGF distribution functions in Europe in 2006.

Europe for the time between 1991 and 2006. Equations (20)(21) are also written in the coordinate system NED. The coordinate system NED ( $x$-north, $y$-east, $z$-down) is used in observatories of http://intermagnet.org/. The velocity of the westward drift is defined for the coordinate system EarthCentered Earth-Fixed (ECEF). The ECEF or conventional terrestrial coordinate system rotates together with the Earth and has its origin at the center of the Earth. The $x$-axis passes through the equator at the prime meridian. The $z$-axis passes through the North Pole. The $y$-axis can be determined by the right-hand rule to be passing through the equator at 90 degrees of longitude.

Thus, the distribution functions in (20)-(21) for NED must be transformed to ECEF by (22) on the Earth's surface. Equation (22),

$$
\vec{B}_{e}=\widehat{I} \vec{B}
$$

is expanded to (A.2) which is in Appendix A. Here, $\widehat{I}$ is an inversion tensor that transforms it from NED to ECEF. The inversion tensor is determined as

$$
|\widehat{I}|=1
$$

and it is expanded to (A.1) in Appendix A.

\section{Solution of the Drift Velocity}

Now, the residual field in (13) is rewritten in the form of (24). And (22) is substituted into (24) which is

$$
\begin{aligned}
\vec{R}_{e}(\vec{r}, t) & =\frac{d \vec{B}_{e}}{d t}+\left(\vec{v}_{0}^{\prime} \cdot \vec{\nabla}_{e}\right) \vec{B}_{e} \\
& =\frac{d(\widehat{I} \vec{B})}{d t}+\left(\vec{v}_{0}^{\prime} \cdot \vec{\nabla}_{e}\right)(\widehat{I} \vec{B}) .
\end{aligned}
$$

We must define the quadrate of the residual field. It is written in (25) which is

$$
\begin{aligned}
{\left[\vec{R}_{e}(\vec{r}, t)\right]^{2} } & =\left[\widehat{I} \frac{d \vec{B}}{d t}+\widehat{I}\left(\vec{v}_{0}^{\prime} \cdot \vec{\nabla}_{e}\right) \vec{B}\right]^{2} \\
& =\widehat{I}^{2}\left[\frac{d \vec{B}}{d t}+\left(\vec{v}_{0}^{\prime} \cdot \vec{\nabla}_{e}\right) \vec{B}\right]^{2},
\end{aligned}
$$

where $\widehat{I}^{2}$ is the square of the inversion tensor. $\widehat{I}^{2}=1 . \vec{\nabla}_{e}$ is Nable's operator in ECEF.

The reason is that quadrate of a vector is not varied by the inversion operation or the rotation of coordinate system on the Earth's surface. Equation (25) can be written as

$$
\left[\vec{R}_{e}(\vec{r}, t)\right]^{2}=\left[\frac{d \vec{B}}{d t}+\left(\vec{v}_{0}^{\prime} \cdot \vec{\nabla}_{e}\right) \vec{B}\right]^{2}
$$

Equation (26) is called the variance of the energy density of the residual field in the small interval of time. Thus, we can define the energy of the residual field via

$$
E=\int\left[\vec{R}_{e}(\vec{r}, t)\right]^{2} d s
$$

where $d s$ is an interval of space and time, $\vec{R}_{e}(\vec{r}, t)$ is the residual field, $\left[\vec{R}_{e}(\vec{r}, t)\right]^{2}$ is the variance of the energy density of the residual field in the small interval of time, and $E$ is the energy of the residual field.

Components of the residual field in (27) are

$$
\begin{aligned}
& R_{e x}(\vec{r}, t)=\vec{i} \frac{d B x}{d t}+\vec{i}\left[v_{0 x}^{\prime} \frac{d B x}{d x}+v_{0 y}^{\prime} \frac{d B x}{d y}+v_{0 z}^{\prime} \frac{d B x}{d z}\right], \\
& R_{e y}(\vec{r}, t)=\vec{j} \frac{d B y}{d t}+\vec{j}\left[v_{0 x}^{\prime} \frac{d B y}{d x}+v_{0 y}^{\prime} \frac{d B y}{d y}+v_{0 z}^{\prime} \frac{d B y}{d z}\right], \\
& R_{e z}(\vec{r}, t)=\vec{k} \frac{d B z}{d t}+\vec{k}\left[v_{0 x}^{\prime} \frac{d B z}{d x}+v_{0 y}^{\prime} \frac{d B z}{d y}+v_{0 z}^{\prime} \frac{d B z}{d z}\right] .
\end{aligned}
$$

When the study of westward drift is done in North America, (20) is substituted into (28).

In the coordinate system ECEF, the components of the drift velocity in (28) are

$$
\begin{aligned}
& v_{0 x}^{\prime}=-r \sin \theta \cos \varphi \cdot \dot{\theta}-r \cos \theta \sin \varphi \cdot \omega, \\
& v_{0 y}^{\prime}=-r \sin \theta \sin \varphi \cdot \dot{\theta}+r \cos \theta \cos \varphi \cdot \omega, \\
& v_{0 z}^{\prime}=r \cos \theta \cdot \dot{\theta} .
\end{aligned}
$$

In case (28) and (29) are substituted into the functional in (27), it becomes

$$
E_{a m}=\int_{1991}^{2006}\left[\int_{0}^{R_{0}} \int_{\pi / 9}^{4 \pi / 9} \int_{10 \pi / 9}^{16 \pi / 9}\left(R_{e x}^{2}+R_{e y}^{2}+R_{e z}^{2}\right) r^{2} \cos \theta d \varphi d \theta d r\right] d t,
$$


where $R_{e x}, R_{e y}$, and $R_{e z}$ are

$$
\begin{aligned}
& R_{e x}=\vec{i}\left(a_{1}+b_{1} \cdot \omega+c_{1} \cdot \dot{\theta}\right), \\
& R_{e y}=\vec{j}\left(a_{2}+b_{2} \cdot \omega+c_{2} \cdot \dot{\theta}\right), \\
& R_{e z}=\vec{k}\left(a_{3}+b_{3} \cdot \omega+c_{3} \cdot \dot{\theta}\right),
\end{aligned}
$$

and where $a_{1}, a_{2}, a_{3}, b_{1}, b_{2}, b_{3}, c_{1}, c_{2}$, and $c_{3}$ are expanded in (A.3) in Appendix A.

We have defined the latitudinal and longitudinal drift velocities from the condition that the energy variation of the residual field is zero in (15).

\section{Results and Discussion}

When the functional in (30) is integrated by the intervals of space and time, it becomes

$$
\begin{aligned}
E_{a m}(\omega, \dot{\theta})=R_{0}^{3}( & m_{1} \dot{\theta}+m_{2} \omega \\
& \left.+m_{3} \dot{\theta} \omega+m_{4} \dot{\theta}^{2}+m_{5} \omega^{2}\right),
\end{aligned}
$$

where $m_{1}, m_{2}, m_{3}, m_{4}$, and $m_{5}$ are the coefficients that are calculated in (B.7) in Appendix B.

Equation (15) can be rewritten in the form

$$
\delta E_{a m}(\omega, \dot{\theta})=\frac{\partial E_{a m}(\omega, \dot{\theta})}{\partial \omega} \delta \omega+\frac{\partial E_{a m}(\omega, \dot{\theta})}{\partial \dot{\theta}} \delta \dot{\theta}=0
$$

From (33), we can write the following condition:

$$
\begin{aligned}
& \frac{\partial E_{a m}(\omega, \dot{\theta})}{\partial \omega}=0, \\
& \frac{\partial E_{a m}(\omega, \dot{\theta})}{\partial \dot{\theta}}=0 .
\end{aligned}
$$

Then, we can also write the following linear equation system:

$$
\begin{aligned}
& m_{2}+m_{3} \dot{\theta}+2 m_{5} \omega=0, \\
& m_{1}+m_{3} \dot{\theta}+2 m_{4} \omega=0 .
\end{aligned}
$$

We can solve the previous linear equation system, and its solutions will be

$$
\begin{aligned}
& \omega=-0.123 \text { degree/year, } \\
& \dot{\theta}=0.068 \text { degree/year. }
\end{aligned}
$$

From the results of this investigation, we see that the longitudinal and latitudinal drifts existed in North America in 19912006.

When the study of westward drift is done for Europe, (21) is substituted into the functional in (30). Moreover, intervals of the integral in (30) are changed as

$$
E_{e u}=\int_{1991}^{2006}\left[\int_{0}^{R_{0}} \int_{\pi / 6}^{4 \pi / 9} \int_{-\pi / 18}^{2 \pi / 9}\left(R_{e x}^{2}+R_{e y}^{2}+R_{e z}^{2}\right) r^{2} \cos \theta d \varphi d \theta d r\right] d t .
$$

After the functional in (37) is integrated by the previous intervals, it becomes

$$
E_{e u}(\omega, \dot{\theta})=R_{0}^{3}\left(n_{1} \dot{\theta}+n_{2} \omega+n_{3} \dot{\theta} \omega+n_{4} \dot{\theta}^{2}+n_{5} \omega^{2}\right),
$$

where $n_{1}, n_{2}, n_{3}, n_{4}$ and $n_{5}$ are coefficients, values of which are calculated in (B.8) in the Appendix B.

The linear equation system

$$
\begin{aligned}
& n_{2}+n_{3} \dot{\theta}+2 n_{5} \omega=0, \\
& n_{1}+n_{3} \dot{\theta}+2 n_{4} \omega=0
\end{aligned}
$$

can be written from the condition like in (33). The solutions of (39) are

$$
\begin{gathered}
\omega=-0.257 \text { degree/year, } \\
\dot{\theta}=7.356 \cdot 10^{-4} \text { degree/year } \approx 0 .
\end{gathered}
$$

Wei and $\mathrm{Xu}[5,7]$ defined the longitudinal and the global mean velocity of westward drift as $-0.18 \mathrm{deg} / \mathrm{yr}$ in MGF for the period 1900-2000. Bullard et al. [3] also defined the mean westward drift as $-0.26 \mathrm{deg} / \mathrm{yr}$ in secular variation by the study for the period 1907-1945.

Their method is based on the spherical harmonic formula for the scalar potential of MGF. And the spherical harmonic formula is the global properties.

MGF distribution functions of space and time can be clearly defined on the little area of Earth's surface. On the little area, MGF distribution functions are more local properties than the spherical harmonic formula. Thus, our method is more accurate than the method of spherical harmonic analyze on the local little areas.

The westward drift in MGF on the Earth's surface is observed by the influence of fluid motions on CMB. Therefore, the properties of fluid motions on $\mathrm{CMB}$ can also be studied, in the opposite way, using the local properties of westward drift on the Earth's surface.

The probability of a dependence of the velocity of fluid motions on the latitude is high.

If we could build MGF distribution functions of space and time on the little areas differed with the latitudes as in Figure 7, we could be able to study the velocity of the fluid motions on the CMB using the velocity of westward drift. If the velocity of westward drift on the Earth's surface depends on the latitude, a similar dependence on the latitude must exist for the velocity of the fluid motions on the CMB. This information is important to the modeling of MGF. These are the advantages of our method.

\section{Conclusions}

The advection term $-\left(\vec{V}_{0} \cdot \vec{\nabla}\right) \vec{B}_{0}$ in (11) is the main factor in the time variation or the secular variation of MGF. In other words, (12) for the CMB very weakly depends on the terms in the right-hand side. And the energy variation does not exist in the strong toroidal field in a few years. They are the theoretical basis of this method to define the westward drift.

The westward drift can also be estimated using this method for the little area of Earth's surface, the MGF 


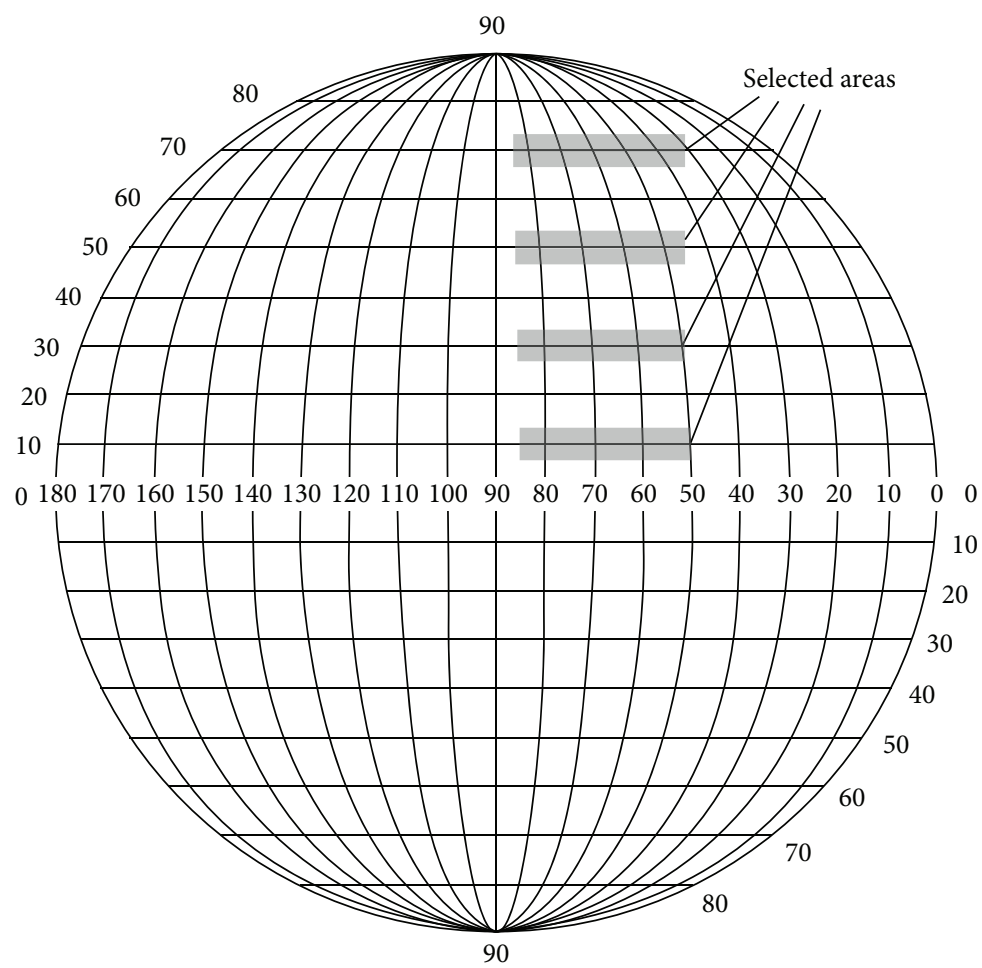

FIGURE 7: Samples of the selected areas.

distribution functions of space and time of which are well known.

In this paper, we have also defined the MGF distribution functions of space and time using the data of observatories located in North America and Europe. And the westward drifts have been estimated in the following forms. The longitudinal -0.123 degree/year and latitudinal 0.068 degree/year drifts existed in North America in 1991-2006. And the longitudinal -0.257 degree/year drift has been defined for Europe in 1991-2006. A latitudinal drift was not Europe in 1991-2006.

The results of these drifts are similar to the results by other studies which used the method of spherical harmonic formula.

\section{Appendices}

\section{A. Mathematics}

Inversion tensor that transforms NED to ECEF is written in the equation

$$
|\widehat{I}|=\left|\begin{array}{ccc}
-\sin \theta \cdot \cos \varphi & -\sin \varphi & -\cos \theta \cdot \cos \varphi \\
-\sin \theta \cdot \sin \varphi & \cos \varphi & -\cos \theta \cdot \sin \varphi \\
\cos \theta & 0 & -\sin \theta
\end{array}\right|=1
$$

where $\theta$ and $\varphi$ are the latitude and longitude in the geodetic coordinates.
Equation (22) is rewritten in the following form:

$$
\left[\begin{array}{l}
B_{x e} \\
B_{y e} \\
B_{z e}
\end{array}\right]=\left|\begin{array}{ccc}
-\sin \theta \cdot \cos \varphi & -\sin \varphi & -\cos \theta \cdot \cos \varphi \\
-\sin \theta \cdot \sin \varphi & \cos \varphi & -\cos \theta \cdot \sin \varphi \\
\cos \theta & 0 & -\sin \theta
\end{array}\right|\left[\begin{array}{l}
B_{x} \\
B_{y} \\
B_{z}
\end{array}\right],
$$

where $B_{x e}$ is the parallel component with $x$ axis of ECEF of geomagnetic fields on the points of the Earth's surface, $B_{y e}$ is the parallel component with $y$ axis of ECEF of geomagnetic fields, and $B_{z e}$ is also the parallel component with $z$ axis of ECEF of geomagnetic field.

The coefficients in (31) are rewritten in the following forms:

$$
\begin{aligned}
& a_{1}=\frac{d B_{x}}{d t}, \quad a_{2}=\frac{d B_{y}}{d t}, \quad a_{3}=\frac{d B_{z}}{d t}, \\
& b_{1}=\frac{d B_{x}}{d y} r \cos \theta \cos \varphi-\frac{d B_{x}}{d x} r \cos \theta \sin \varphi, \\
& b_{2}=\frac{d B_{y}}{d y} r \cos \theta \cos \varphi-\frac{d B_{y}}{d x} r \cos \theta \sin \varphi, \\
& b_{3}=\frac{d B_{z}}{d y} r \cos \theta \cos \varphi-\frac{d B_{z}}{d x} r \cos \theta \sin \varphi, \\
& c_{1}=\frac{d B_{x}}{d z} r \cos \theta-\frac{d B_{x}}{d y} r \sin \theta \sin \varphi-\frac{d B_{x}}{d x} r \sin \theta \cos \varphi,
\end{aligned}
$$




$$
\begin{array}{ll}
c_{2}=\frac{d B_{y}}{d z} r \cos \theta-\frac{d B_{y}}{d y} r \sin \theta \sin \varphi-\frac{d B_{y}}{d x} r \sin \theta \cos \varphi, & \frac{d \theta}{d y}=-\frac{\sin \varphi \sin \theta}{r}, \\
c_{3}=\frac{d B_{z}}{d z} r \cos \theta-\frac{d B_{z}}{d y} r \sin \theta \sin \varphi-\frac{d B_{z}}{d x} r \sin \theta \cos \varphi . & \frac{d \theta}{d z}=\frac{\cos \theta}{r} .
\end{array}
$$

And the derivatives in (A.3) could be written in the following forms:

$$
\begin{aligned}
& \frac{d B_{x}}{d x}=\frac{d B_{x}}{d \varphi} \frac{d \varphi}{d x}+\frac{d B_{x}}{d \theta} \frac{d \theta}{d x} \\
& \frac{d B_{y}}{d x}=\frac{d B_{y}}{d \varphi} \frac{d \varphi}{d x}+\frac{d B_{y}}{d \theta} \frac{d \theta}{d x} \\
& \frac{d B_{z}}{d x}=\frac{d B_{z}}{d \varphi} \frac{d \varphi}{d x}+\frac{d B_{z}}{d \theta} \frac{d \theta}{d x} \\
& \frac{d B_{x}}{d y}=\frac{d B_{x}}{d \varphi} \frac{d \varphi}{d y}+\frac{d B_{x}}{d \theta} \frac{d \theta}{d y} \\
& \frac{d B_{y}}{d y}=\frac{d B_{y}}{d \varphi} \frac{d \varphi}{d y}+\frac{d B_{y}}{d \theta} \frac{d \theta}{d y} \\
& \frac{d B_{z}}{d y}=\frac{d B_{z}}{d \varphi} \frac{d \varphi}{d y}+\frac{d B_{z}}{d \theta} \frac{d \theta}{d y} \\
& \frac{d B_{x}}{d z}=\frac{d B_{x}}{d \varphi} \frac{d \varphi}{d z}+\frac{d B_{x}}{d \theta} \frac{d \theta}{d z} \\
& \frac{d B_{y}}{d z}=\frac{d B_{y}}{d \varphi} \frac{d \varphi}{d z}+\frac{d B_{y}}{d \theta} \frac{d \theta}{d z} \\
& \frac{d B_{z}}{d \varphi} \frac{d \varphi}{d z}+\frac{d B_{z}}{d \theta} \frac{d \theta}{d z}
\end{aligned}
$$

Now, it is easy to define the derivatives $d B_{x} / d x$, $d B_{y} / d x, d B_{z} / d x, d B_{x} / d y, d B_{y} / d y, d B_{z} / d y, d B_{x} / d z$, $d B_{y} / d z$, and $d B_{z} / d z$ using the equations

$$
\begin{aligned}
& \frac{d \varphi}{d x}=\frac{d}{d x} \arccos \left(\frac{x}{\sqrt{x^{2}+y^{2}}}\right)=-\frac{\sin \varphi}{r \cos \theta}, \\
& \frac{d \varphi}{d y}=\frac{\cos \varphi}{r \cos \theta}, \\
& \frac{d \varphi}{d z}=0 \\
& \frac{d \theta}{d x}=\frac{d}{d x} \arccos \left(\frac{\sqrt{x^{2}+y^{2}}}{\sqrt{x^{2}+y^{2}+z^{2}}}\right)=-\frac{\cos \varphi \sin \theta}{r},
\end{aligned}
$$

For example, $d B_{x} / d x$ can be written like

$$
\begin{aligned}
\frac{d B_{x}}{d x}=- & \frac{\sin \varphi}{r \cos \theta}\left(C_{01}+2 C_{02} \varphi+3 C_{03} \varphi^{2}\right. \\
& \left.+C_{11} \theta+2 C_{12} \theta \varphi+C_{21} \theta^{2}\right) \\
-\frac{\cos \varphi \sin \theta}{r}( & C_{10}+2 C_{20} \theta+3 C_{30} \theta^{2} \\
& \left.+C_{11} \varphi+2 C_{21} \varphi \theta+C_{12} \varphi^{2}\right) .
\end{aligned}
$$

\section{B. Calculations}

Our calculation should be as clear as possible. Thus, we have done some normalization in the scales of time and coordinate. When we define the coefficients in (20)-(21), we have changed the scale of time for 1991-2006 years by the 1-16 years. And the $\varphi$ longitude is changed by $\varphi-180$ only in North America. The values of westward drift must not be changed in the previous normalization. The normalization has only reduced the order of the polynomial coefficients in (20)-(21). And it helps to make the estimation clear.

In North America, the following polynomial coefficients have been defined for the longitudinal scale $\varphi-180$ and the time scale of 1-16 years. And in Europe, the polynomial coefficients are also defined for the time interval of 116 years. Thus, we have integrated (30) and (37) by the previous intervals of time and coordinate in North America and Europe. But these are the technical problems in the calculation. Therefore we do not change the intervals of time and longitude in (30) and (37), because (30) and (37) should be understandable for researchers.

The dependences of the coefficients in (20) and (21) on time are defined as

$$
\begin{aligned}
& C_{00}=-19.375 t^{2}+898.247 t+32074.697, \\
& C_{01}=27.286 t^{2}-1319.913 t-9026.928, \\
& C_{02}=-10.194 t^{2}+595.632 t+1691.978, \\
& C_{03}=0.652 t^{2}-78.083 t-91.66, \\
& C_{10}=-1065.704 t+52252.804, \\
& C_{11}=-17.426 t^{2}+962.015 t-14691.562, \\
& C_{12}=5.645 t^{2}-222.753 t+5722.045, \\
& C_{20}=-3.849 t^{3}+108.95 t^{2}+115.72 t-89763.49,
\end{aligned}
$$


$C_{21}=-186.744 t+410.167$

$C_{30}=1.637 t^{3}-46.433 t^{2}+68.74 t+33421.397 ;$

$A_{00}=16.855 t^{2}-0.913 t-16101.254$,

$A_{01}=1.395 t^{3}-60.858 t^{2}+204.93 t+54636.953$,

$A_{02}=-0.664 t^{3}+29.015 t^{2}-121.327 t-39358.43$,

$A_{03}=0.125 t^{3}-5.309 t^{2}+30.47 t+7480.44$,

$A_{10}=-14.693 t^{2}-220.23 t+25874.22$,

$A_{11}=15.659 t^{2}+168.957 t-26226.375$,

$A_{12}=-1.58 t^{2}-25.624 t+5391.304$,

$A_{20}=170.34 t-16502.614$,

$A_{21}=-5.76 t^{2}-32.61 t+9350.964$,

$A_{30}=-0.443 t^{3}+14.337 t^{2}-126.78 t+1100.061 ;$

$K_{00}=-3.697 t^{3}+145.03 t^{2}-878.612 t-109655.43$,

$K_{01}=3.29 t^{3}-142.08 t^{2}+1220.517 t+132037.83$,

$K_{02}=-0.882 t^{3}+44.47 t^{2}-579.29 t-37867.043$,

$K_{03}=-1.98 t^{2}+51.055 t+401.07$,

$K_{10}=6.912 t^{3}-243.205 t^{2}+955.721 t+206508.148$,

$K_{11}=-3.075 t^{3}+120.14 t^{2}-769.72 t-113743.611$,

$K_{12}=0.99 t^{3}-36.184 t^{2}+418.863 t+25710.492$,

$K_{20}=-5.42 t^{3}+174.353 t^{2}-638.703 t-55351.172$,

$K_{21}=-299.78 t+14775.592$,

$K_{30}=2.29 t^{3}-68.197 t^{2}+490.495 t-5461.943 ;$

$D_{00}=-0.79 t^{3}+28.22 t^{2}-253.338 t+49320.938$,

$D_{01}=-290.1645 t+6472.407$,

$D_{02}=212.34 t+5017.157$,

$D_{03}=-0.0484 t^{3}-1.4166 t^{2}+34.979 t-681.798$,

$D_{10}=2.612 t^{3}-91.474 t^{2}+922.318 t-38396.7694$,

$D_{11}=453.385 t-16664.086$,

$D_{12}=-191.376 t-6551.478$,

$D_{20}=-2.751 t^{3}+95.569 t^{2}-1006.358 t+5209.597$,

$D_{21}=-200.728 t+10926.704$,

$D_{30}=0.945 t^{3}-32.644 t^{2}+349.745 t+73.998 ;$

(B.4)
$Q_{00}=-0.601 t^{3}+24.324 t^{2}-269.764 t+1814.066$,

$Q_{01}=-1.122 t^{3}+32.228 t^{2}-162.816 t+4613.38$,

(B.1)

$Q_{02}=-6.53 t^{2}+315.947 t-16129.512$,

$Q_{03}=-2.482 t^{2}+96.678 t+655.054$,

$Q_{10}=2.222 t^{3}-85.493 t^{2}+1037.27 t-7733.423$,

$Q_{11}=-446.872 t+11705.439$,

$Q_{12}=-228.862 t+12840.865$,

$Q_{20}=-2.72 t^{3}+98.783 t^{2}-1096.699 t+5678.082$,

$Q_{21}=304.279 t-8005.954$,

$Q_{30}=1.059 t^{3}-36.481 t^{2}+366.018 t-1303.48 ;$

$H_{00}=-2.811 t^{3}+86.553 t^{2}-730.614 t-42252.381$,

$H_{01}=-4.596 t^{3}+140.1074 t^{2}-833.826 t-1043.21$,

$H_{02}=33.781 t^{2}-1224.008 t+15682.71$,

$H_{03}=-5.79 t^{2}+148.61 t-3773.1026$

$H_{10}=9.595 t^{3}-308.561 t^{2}+2816.664 t+192757.05$,

$H_{11}=9.245 t^{3}-298.329 t^{2}+2267.262 t+8822.885$,

$H_{12}=-1.882 t^{3}+16.501 t^{2}+777.267 t-7568.86$,

(B.3) $\quad H_{20}=-10.759 t^{3}+357.114 t^{2}-3361.87 t-142531.088$,

$H_{21}=-4.553 t^{3}+156.063 t^{2}-1390.52 t-7658.873$,

$H_{30}=3.912 t^{3}-132.968 t^{2}+1283.618 t+38877.982$.

The values of the coefficients in (35) and (38) are calculated as follows:

$$
\begin{aligned}
& m_{1}=-32357481.6, \\
& m_{2}=7677960.7 \\
& m_{3}=2726671352.51, \\
& m_{4}=16031093418.8, \\
& m_{5}=2597509055.6 ; \\
& n_{1}=2751704.38, \\
& n_{2}=1117572.227, \\
& n_{3}=645280739.23,
\end{aligned}
$$




$$
\begin{aligned}
& n_{4}=5626256620.658, \\
& n_{5}=125423203.758 .
\end{aligned}
$$

\section{References}

[1] E. Halley, "On the cause of the change in the variation of the magnetic needle, with an hypothesis of the structure of the internal parts of the earth," Philosophical Transactions of the Royal Society of London, vol. 17, pp. 470-478, 1692.

[2] L. A. Bauer, "On the secular motion of a free magnetic needle," Physical Review (Series I), vol. 2, pp. 455-465, 1895.

[3] E. C. Bullard, C. Freedman, H. Gellman, and J. Nexon, "The westward drift of the Earth's magnetic field," Philosophical Transactions of the Royal Society A, vol. 243, pp. 67-92, 1950.

[4] T. Yukutake, "The westward drift of the magnetic field of the Earth," Bulletin of the Earthquake Research Institute, vol. 40, pp. $1-65,1962$.

[5] Z. Wei and W. Xu, "Latitude-dependence and dispersion of the westward drift in the geomagnetic field," Chinese Science Bulletin, vol. 47, no. 4, pp. 330-333, 2002.

[6] J. M. Herndon, "Nature of planetary matter and magnetic field generation in the solar system," Current Science, vol. 96, no. 8, pp. 1033-1039, 2009.

[7] Z. Wei and W. Xu, "Differential rotation of geomagnetic field," Chinese Science Bulletin, vol. 48, no. 24, pp. 2739-2742, 2003.

[8] T. Yukutake and H. Tachinaka, "The westward drift of the geomagnetic secular variation," Bulletin of the Earthquake Research Institute, vol. 46, pp. 1075-1102, 1968. 

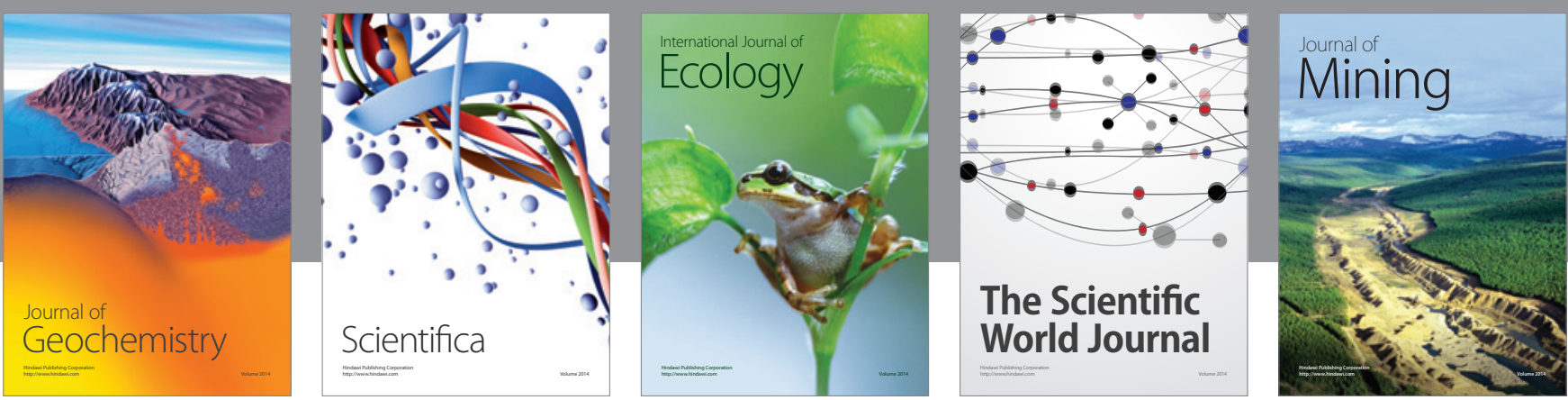

The Scientific World Journal
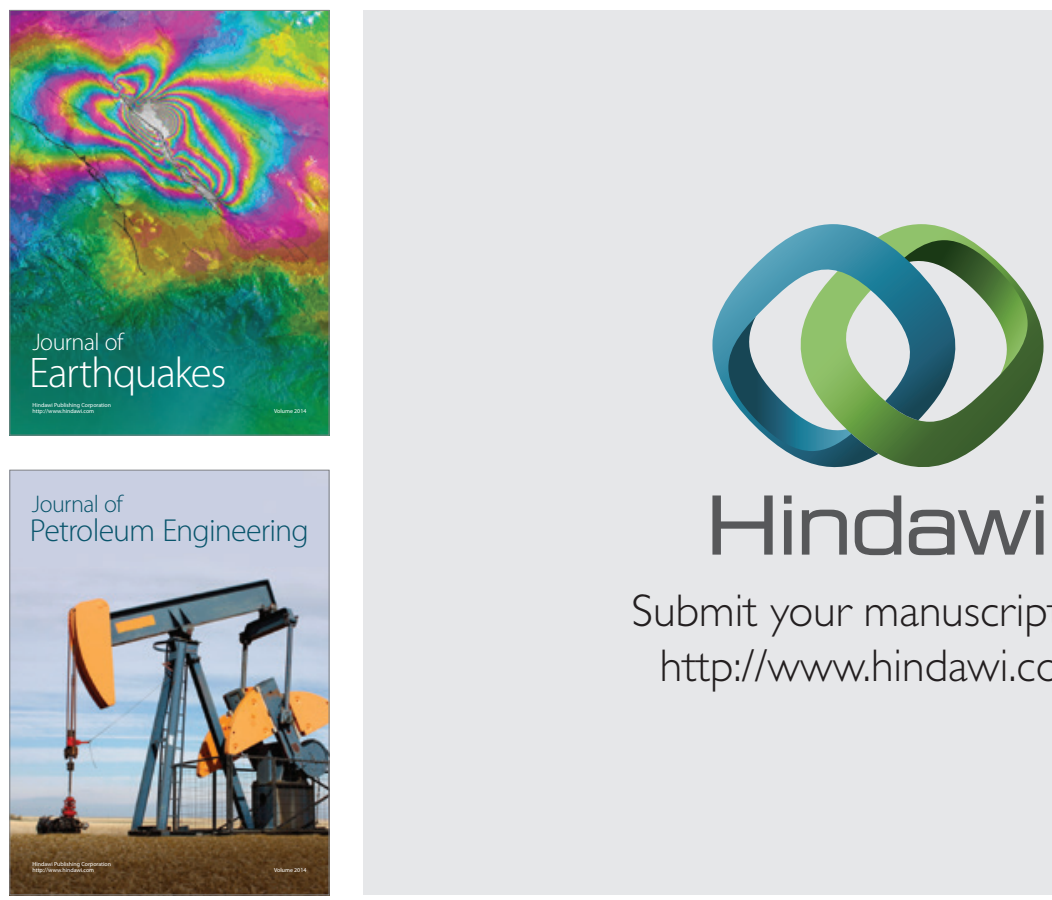

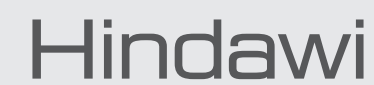

Submit your manuscripts at

http://www.hindawi.com
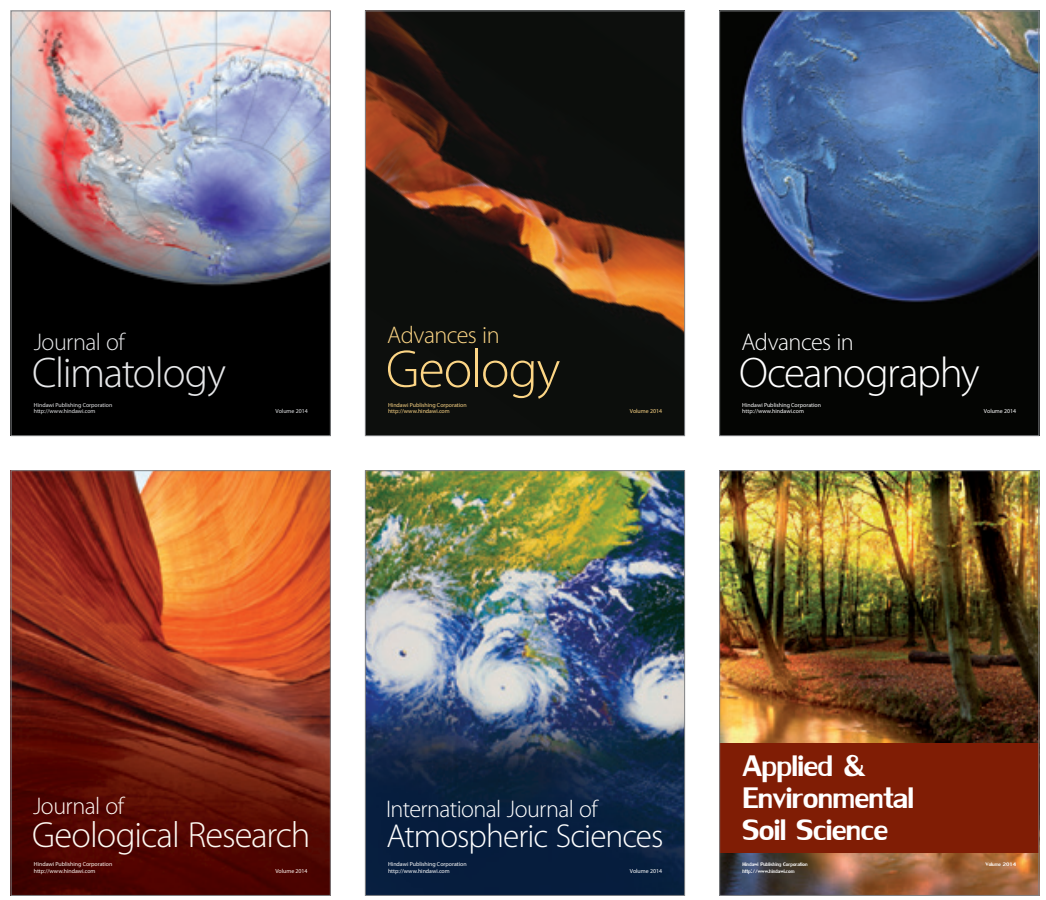
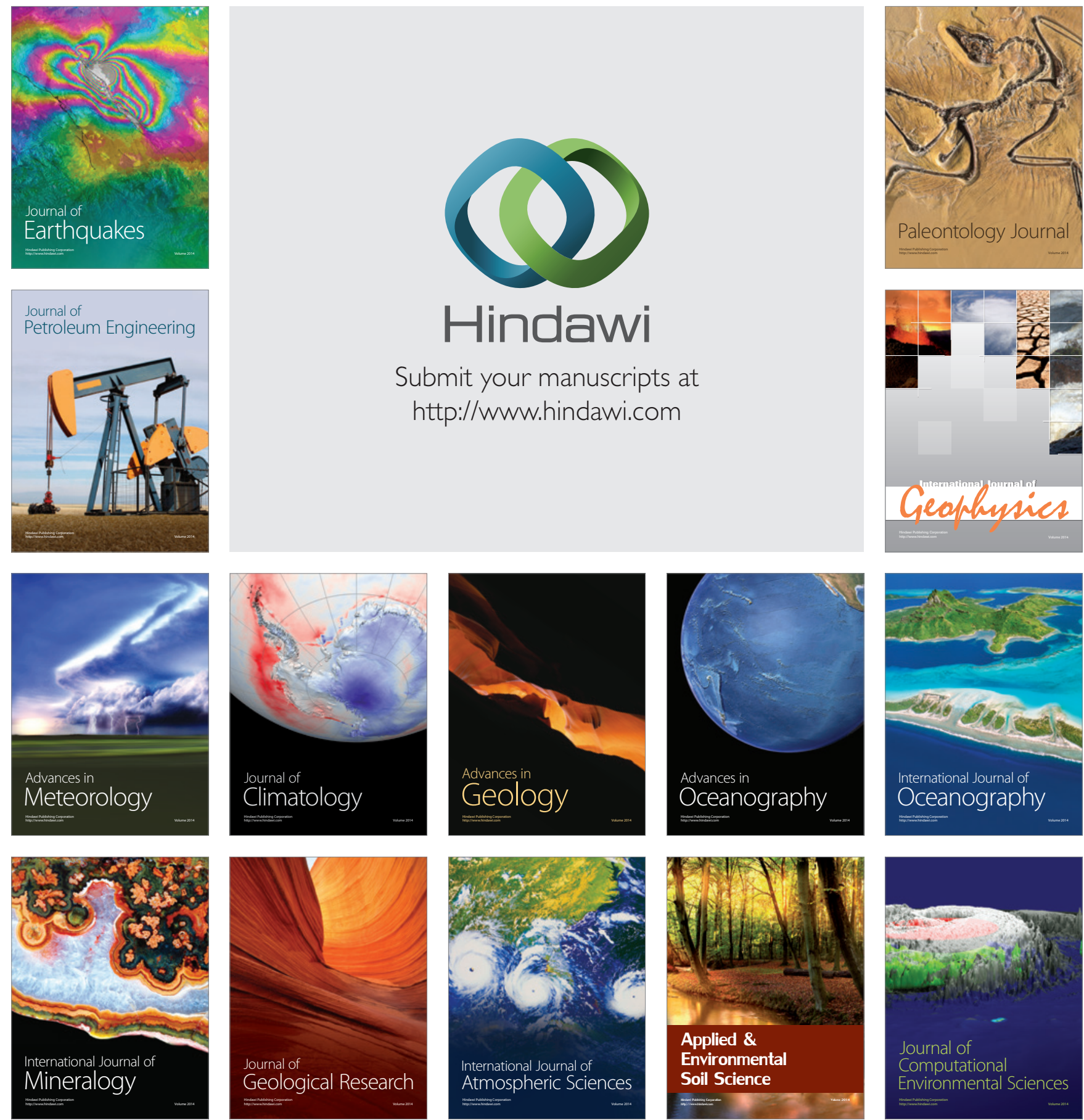